\title{
Current state of interventional radiotherapy (brachytherapy) education in Italy: results of the INTERACTS survey
}

Luca Tagliaferri, MD, PhD! György Kovács, MD, PhD², Cynthia Aristei, MD³ , Vitaliana De Sanctis, MD, Fernando Barbera, MD5 , Alessio Giuseppe Morganti, MD6. Calogero Casà, MD77, Bradley Rumwell Pieters, MD, PhD8 Elvio Russi, MD9, Lorenzo Livi, MD10, Renzo Corvò, MD"l, Andrea Giovagnoni, MD², Umberto Ricardi, MD'13, Vincenzo Valentini, MD ${ }^{14,15}$, Stefano Maria Magrini, MD'b and the Directors of the Italian Radiation Oncology Schools* IChair of the Brachytherapy, Interventional Radiotherapy and IORT Study Group of the Italian Radiotherapy and Clinical Oncology Society (AIRO); Gemelli ART (Advanced Radiation Therapy), UOC Radioterapia, Fondazione Policlinico Universitario A. Gemelli IRCCS, Rome, Italy, 2INTERACTS (Interventional Radiotherapy Active Teaching School) Educational Program Director; Head, Interdisciplinary Brachytherapy Unit, University of Lübeck/UKSH CL, Lübeck, Germany, ${ }^{3}$ Past Chair of AIRO Brachytherapy study group; Head, Radiation Oncology Section, Department of Surgery and Biomedical Sciences, University of Perugia and Perugia General Hospital, Perugia, Italy, ${ }^{4}$ Deputy Chair of the Brachytherapy, Interventional Radiotherapy and IORT AIRO Study Group; Department of Radiation Oncology, Faculty of Medicina e Psicologia, Sant'Andrea Hospital, University of Rome "La Sapienza", Rome, Italy. 'Board Member of the Brachytherapy, Interventional Radiotherapy and IORT AIRO Study Group; Head, Brachytherapy Section, Radiation Oncology Department, Spedali Civili Hospital and Brescia University, Brescia, Italy, 'Member of the Scientific Committee and Teacher, INTERACTS School; Head, Radiation Oncology Center. Department of Experimental, Diagnostic and Specialty Medicine (DIMES), S. Orsola-Malpighi Hospital, University of Bologna, Bologna, Italy, ${ }^{7}$ National Coordinator of Resident Physician Department (SIMS) of the Italian Young Doctors Association (SIGM); Istituto di Radiologia. Università Cattolica del Sacro Cuore, Rome, Italy, ${ }^{8}$ Amsterdam UMC, University of Amsterdam, Department of Radiation Oncology, Cancer Center Amsterdam, The Netherlands, ${ }^{9}$ AlRO Past President; Head, Radiotherapy Unit, Ospedale S. Croce e Carle, Cuneo, Italy, ${ }^{10}$ Chair of the Section for Radiation Oncology Schools, National College of Professors of Radiology, Radiotherapy and Nuclear Medicine; Head, Radiation Oncology Unit, Azienda Ospedaliera Universitaria Careggi, University of Florence, Florence, Italy, "Chair of the Scientific Commission, AlRO; Head, Radiation Oncology Department, IRCCS San Martino-IST, National Cancer Research Institute, University of Genoa, Genoa, Italy, ${ }^{12}$ Chair of the National College of Professors of Radiology, Radiotherapy and Nuclear Medicine; Head, Radiology Department, University of Ancona, Ancona, Italy, ${ }^{13}$ Chair of the Department of Oncology; Head, Radiation Oncology Department, University of Turin, Turin, Italy, ${ }^{14} \mathrm{Head}$, Gemelli ART (Advanced Radiation Therapy) - UOC Radioterapia - Fondazione Policlinico Universitario A. Gemelli IRCCS, Rome, Italy, ${ }^{15}$ INTERACTS School Director; Università Cattolica del Sacro Cuore, Rome, Italy, ${ }^{16}$ AlRO President; Chairman, Radiation Oncology Section, National College of Professors of Radiology, Radiotherapy and Nuclear Medicine; Head, Radiation Oncology Department, Spedali Civili Hospital and Brescia University, Brescia, Italy

**Directors of the Italian Radiation Oncology Schools

Prof. Alessio Giuseppe Morganti (University of Bologna, Italy). Prof. Stefano Maria Magrini (University of Brescia, Italy), Prof. Lorenzo Livi (University of Firenze, Italy), Prof. Renzo Corvo' (University of Genova, Italy), Prof. Ernesto Di Cesare (University of L'Aquila, Italy), Prof. Stefano Pergolizzi (University of Messina, Italy). Prof. Barbara Jereczek Fossa (University of Milano, Italy). Prof. Gianstefano Gardani (University of Milano Bicocca, Italy), Prof. Roberto Pacelli (University of Napoli - "Federico Il", Italy), Prof. Salvatore Cappabianca (University of Napoli - "Vanvitelli", Italy), Prof. Marco Krengli (University of Novara, Italy), Prof. Fabio Pomerri (University of Padova, Italy), Prof. Antonio Lo Casto (University of Palermo, Italy), Prof. Cynthia Aristei (University of Perugia, Italy), Prof. Fabiola Paiar (University of Pisa, Italy), Prof. Vincenzo Tombolini (University of Roma - La Sapienza I, Italy), Prof. Francesco Scopinaro (University of Roma - La Sapienza II, Italy), Prof. Riccardo Santoni (University of Roma - "Tor Vergata”, Italy), Prof. Sara Ramella (Campus Biomedico University, Rome, Italy), Prof. Vincenzo Valentini (Catholic University, Rome, Italy), Prof. Angela Spanu (University of Sassari, Italy), Prof. Luigi Pirtoli (University of Siena, Italy), Prof. Umberto Ricardi, (University of Turin, Italy).

\footnotetext{
Address for correspondence: Luca Tagliaferri, MD, PhD, UOC Radioterapia - Fondazione Policlinico Universitario A. Gemelli IRCCS - Largo A. Gemelli, 8 - 00168 - Rome, Italy, 


\begin{abstract}
Purpose: Increased complexity of interventional radiotherapy (brachytherapy - BT) treatment planning and quality control procedures has led to the need of a specific training. However, the details of the features of BT learning objectives and their distribution in the training paths of the Italian Radiation Oncology Schools are not known. This paper aims to provide the actual 'state-of-the-art' of BT education in Italy and to stimulate the debate on this issue.

Material and methods: All the Italian radiation oncology schools' directors (SD) were involved in a web survey, which included questions on the teaching of BT, considering also the 2011 ESTRO core curriculum criteria. The survey preliminary results were discussed at the $8^{\text {th }}$ Rome INTER-MEETING (INTERventional Radiotherapy Multidisciplinary Meeting), June $24^{\text {th }}$, 2017. The present paper describes the final results of the survey and possible future teaching strategies resulting from the discussion.

Results: A total of 23 SDs answered the survey. The results evidenced a wide heterogeneity in the learning activities available to trainees in BT across the country. While theoretical knowledge is adequately and homogeneously transmitted to trainees, the types of practice to which they are exposed varies significantly among different schools.

Conclusions: This survey proves the need for an improvement of practical BT education in Italy and the advisability of a national BT education programme networking schools of different Universities. Beside the organization of national/international courses for BT practical teaching, Universities may also establish post-specialization courses ('second level' Masters) to allow professionals (already certified in radiation oncology) to acquire more advanced BT knowledge.

Key words: brachytherapy, teaching, interventional radiotherapy, survey.

\section{Purpose}

Rapid evolution and improvement of technology in radiation oncology and the complexity of each area of radiotherapy (RT) practice determined the need for an adequate and flexible practical training system. In the field of interventional radiotherapy (brachytherapy - BT), a decline in BT utilization was detected in the 1990's, essentially due to technological improvement of external beam RT. However, since 2004, a new increase in BT treatments was observed, possibly due to developments in BT planning and delivery $[1,2]$.

Table 1. Five questions on general issue and their possible answers

1. Regarding BT teaching and evaluation, teaching is mostly carried out by (more than one answer): faculty radiation oncologists (ROs), faculty ROs specialist in BT, physicists, fellows, senior residents, outside experts

2. The most common forms of BT teaching are (more than one answer): informal lectures, didactic lectures, problem-based approaches, senior resident presentations, simulation, on site teaching, on site teaching dosimetry, courses

3. Your center has defined specific written objectives with respect to knowledge, skills, and required attitudes for a resident to be competent in BT? Yes (based on ESTRO core curriculum), No

4. Formal evaluations of practical skills are mostly carried out by (more than one answers): direct observation, oral examinations, written examinations, no formal evaluation

5. The main barriers to BT teaching are: lack of clinical practice, lack of time, other
An increased level of complexity in BT treatment planning and quality control procedures has led to the need of specific training. To promote high-level and standardized training, the European Society for Radiotherapy and Oncology (ESTRO) included high-level knowledge of BT in the ESTRO core 2011 curricula [3]. Recently, the Italian Association of Radiation Oncology (AIRO) has published the results of a survey regarding the availability of BT equipment and the type of services offered in the various Italian hospitals [4]. However, it seems useful to understand also the 'state-of-the-art' about training in BT during residency, especially because, in Italian legislation, BT practical teaching is included among the minimum requirements for the accreditation of radiation oncology schools $[5,6]$. However, the features of BT learning objectives and their distribution in the training paths of Italian radiation oncology schools are not known in detail in the Italian context. Therefore, this paper aims to provide some information about the actual 'state-of-the-art' of BT education in Italy, in the light of the 2011 ESTRO core curricula, and to stimulate the debate on this issue. It was realized within the frame of the activities of the INTErventional Radiotherapy ACtive Teaching School (INTERACTS), which declare its mission in the interventional RT education and research $[7,8]$.

\section{Material and methods}

All the Italian radiation oncology schools' directors (SD) were involved in a web survey, which considered the suggestions of the 2011 ESTRO core curricula criteria [3]. To achieve maximum participation, the survey was distributed among the participants in two rounds (June 2017 and Febru- 
Table 2. A question on the level of skills acquisition based on ESTRO core curriculum. Possible answers: very low, low, intermediate, good, very good

1. Identify the technique to be used

2. Identify the patient in advance and be familiar with their general condition

3. Evaluate the treatment plan

4. Identify the roles and responsibilities of the members of the team

5. Recognize the specific radiation hazards associated with brachytherapy

6. Identify the routine quality assurance (QA) and safety procedures that should be carried out prior to treatment

7. Arrange the treatment room and ensure all equipment is available

8. Assemble all the documents required

9. Inform the patient of the procedure

10. If appropriate, participate in planning the treatment

11. Manage the aspects of the procedures that are the responsibility of the RT

12. Check all radiation protection requirements have been met

13. Carry out the routine $Q A$ and safety procedures prior to treatment

14. Ability to recognize and perform combined treatments with EBRT

15. Ability to organize and perform interdisciplinary treatments

ary 2018). As the survey was blinded, in the second submission, we requested the SDs who had already responded to the first submission not to reply again. The survey contained seven questions in English and was hosted by SurveyMon- key (www.surveymonkey.com). The names of respondents were blinded to the authors of this paper.

Five questions (Table 1) were on general issues; one (including multiple items) considered the specific skills the resident should gain (Table 2). One key question aimed at acquiring information on the availability of the different BT procedures in the academic hospital where the school was based or in other structures within the school's network. Respondents were also asked to declare which procedures, in their opinion, should be a part of the resident's training. The listed anatomical disease sites treated with BT included gynecological area, prostate (high-dose-rate and low-doserate), skin, breast, esophagus, head \& neck site, lung (endobronchial), different sites of soft tissue sarcoma, eye, rectum, anus as well as different sites of BT in children.

The survey preliminary results were discussed on $24^{\text {th }}$ June 2017 in a dedicated session of the $8^{\text {th }}$ ROME INTER-MEETING (ROME INTErventional Radiotherapy Multidisciplinary Meeting). The present paper describes the results of the survey and future strategies resulting from the discussion.

\section{Results}

In June 2017, they were 25 of Italian radiotherapy schools, while in February 2018 - 23 schools. A total of 23 SDs responded to the survey.

The questionnaire results showed that BT teaching is mainly done by board certified ROs, experienced and actively practicing BT $(76 \%, 16$ out of 21 responders, 2 responders skipped the question) and to a lesser extent by other faculty ROs ( $57 \%, 12$ responders), physicist ( $38 \%, 8$ responders) as well as occasionally senior residents or invited external experts were involved in brachytherapy education.

Table 3. Brachytherapy (BT) procedures in radiotherapy school

\begin{tabular}{|c|c|c|c|}
\hline BT procedures & $\begin{array}{l}\text { Responders: available in the academic } \\
\text { hospital or in the network } \\
\text { (\% of responders to the question) } \\
\text { [\% of the total participants to the survey] }\end{array}$ & $\begin{array}{l}\text { Responders: should be part of resident's } \\
\text { training } \\
\text { (\% of responders to the question) } \\
\text { [\% of the total participants to the survey] }\end{array}$ & $\begin{array}{l}\text { Total no. of } \\
\text { responders }\end{array}$ \\
\hline Gynecologic & $21(95 \%)[91 \%]$ & $13(59 \%)[57 \%]$ & 22 \\
\hline Prostate HDR & $4(25 \%)[17 \%]$ & 15 (94\%) [65\%] & 16 \\
\hline Prostate LDR & $6(35 \%)[26 \%]$ & $13(77 \%)$ [57\%] & 17 \\
\hline Skin & $14(82 \%)$ [61\%] & 9 (53\%) [39\%] & 17 \\
\hline Breast & $8(62 \%)[35 \%]$ & $8(62 \%)[35 \%]$ & 13 \\
\hline Esophagus & 9 (69\%) [39\%] & 8 (62\%) [35\%] & 13 \\
\hline Head and neck & 8 (57\%) [35\%] & 9 (64\%) [39\%] & 14 \\
\hline Lung (endobronchial) & $8(80 \%)[35 \%]$ & $5(50 \%)[22 \%]$ & 10 \\
\hline Sarcoma & 9 (53\%) [39\%] & $12(71 \%)$ [52\%] & 17 \\
\hline Eye & $9(60 \%)[39 \%]$ & 8 (53\%) [35\%] & 15 \\
\hline Rectal & $7(54 \%)[30 \%]$ & 7 (54\%) [30\%] & 13 \\
\hline Anus & $12(75 \%)$ [52\%] & $10(63 \%)[43 \%]$ & 16 \\
\hline BT in children & $6(60 \%)[26 \%]$ & $5(50 \%)[22 \%]$ & 10 \\
\hline
\end{tabular}


The most common types of BT education are: on site teaching $(69 \%, 15$ out of 22 responders), didactic lectures $(63 \%, 14$ responders). Further modalities are: problem-based approaches $(55 \%, 12$ responders), on site dosimetry teaching $(46 \%, 10$ responders), informal lectures (46\%, 10 responders), case simulations $(27 \%, 6$ responders), courses (19\%, 4 responders), and senior residents' presentations (19\%, 4 responders).

About half (11 responders out of 23) of Italian residential schools have written specific objectives with respect to knowledge, skills, and required attitudes for a resident to be competent in BT. Three responders $(13 \%)$ had these objectives specifically based on ESTRO core curriculum, and 5 additional responders (22\%) were willing to rewrite the document according to the ESTRO core curriculum.

The evaluation of practical skills of a resident was mostly carried out by oral examination $(74 \%, 17$ responders) or direct observation $(65 \%, 15$ responders), while sporadic cases of no formal evaluation $(17 \%, 4$ responders) and no written examinations were reported.

The main barriers to BT education identified by SD were lack of clinical practice $(63 \%, 12$ responders), with a minority of SDs indicating lack of time as the reason (31\%, 6 responders). Four SDs skipped the question and one $(5 \%)$ answered that there are no main barriers.

Almost all the skills indicated in ESTRO core curriculum and listed in Table 2 were considered important and were acquired at an intermediate or higher level by the resident students.

A question investigated which BT procedures are available and which of these should be part of the resident's training, according to SDs. All Italian radiotherapy schools offer BT procedures and training, but there is a wide heterogeneity. Details are shown in Table 3.

\section{Discussion}

The use of interventional radiotherapy (BT) has increased in the last decades, most probably due to technical improvements, allowing to deliver higher doses to the target while reducing dose and volume of organs at risk [9]. Moreover, BT in Italy has been increasingly based on a multidisciplinary approach, with a growing number of high-quality publications of excellent treatment results $[9,10,11,12,13,14,15,16,17,18,19,20,21,22,23,24,25,26$, $27,28,29,30,31]$. These innovations, in the context of a rapid diffusion of $3 \mathrm{D}$ planning, with an increased use of computed tomography (CT), magnetic resonance imaging (MRI), positron emission tomography (PET)/CT, and ultrasound, favored the diffusion of image-guided brachytherapy (IGBT) [32] with related clinical benefits. The industry also played a central role in this process with the development of miniaturized sources (remote after loading HDR and PDR devices), and even smaller and better manageable applicators, CT/MRI-compatible [2]. The diffusion of dwelling devices paved the way to better treatment plans optimization (for both target coverage and organs at risk [OARs] dose reduction). Intensity modulated brachytherapy has become a reality [33]. These developments have led to an increased accuracy of the treatment and to the development of complex im- age-based control systems of implant stability [2]. Better medical and physical quality assurance protocols have then been developed [34]. The use of intensity-modulated brachytherapy (IMBT) supported by IGBT has led to interesting clinical benefits, in terms of increased local control and decrease in toxicity [35,36,37,38,39,40,41,42, $43,44]$. On the other hand, the need to perform complex IGBT treatments has stimulated additional radiobiology research to introduce hypo-fractionated therapeutic protocols $[2,45]$. The introduction of 'image fusion' systems, self-control, dose optimization, and automatic catheter reconstruction represents another significant progress in planning procedures [46,47].

Analyzing the different kinds of BT procedures/sites treated, three major groups can be identified according to the survey results:

1. Available and important for residential training (gynecologic, skin, anus, breast, esophagus, eye); the percentage of respondents perceiving the need for practical training $(60-95 \%)$ is inferior or equals to the availability in the school network;

2. Important but not sufficiently available (prostate, sarcoma, head and neck); the percentage of respondents perceiving the need for practical training (53-94\%) is superior or equals to the availability in the school network (this is particularly evident in case of prostate cancer);

3. Apparently less relevant for residential training (lung, BT in children, and rectal).

To summarize, BT offers an important contribution in oncology for a variety of tumor sites [48]. Thus, the knowledge of basic principles, indications, limiters, and possible integrations of BT with other treatment modalities should be an important part of the core curriculum of RO's. The ESTRO core curriculum offers a good guidance to standardization and formalization of contents, but the main obstacle to set up an effective and high-level training in interventional BT is the lack of actual clinical practice. The central role of personal experience and procedural observation has been highlighted during the meeting. Due to the importance of practical skills requested by BT practice, a theoretical teaching could only partially replace on-site education. Direct observation of BT is the best way to learn indications/contraindications as well as procedures. The use of simulation or dummies can be another possibility of training, especially for rarer procedures. Although not all the board-certified ROs will practice BT in their professional life, all of them should be aware when a patient profits from an interventional RT treatment.

The present survey has some drawbacks: a) realized in two phases, b) obtained answers to the different questions from a variable number of respondents. However, the present status of BT teaching seems to clearly emerge from the data collected and herein presented.

In fact, the present survey has evidenced a wide heterogeneity in practical learning activities available to trainees in radiation oncology across the country. Even if theoretical teaching is guaranteed, the types of practice to which trainees are exposed vary significantly among different schools. This is the reason why the survey re- 
sults have emphasized the need of standardization of the training system through the adoption of unified core curriculum. Italian law recently introduced an accreditation system, enforcing strict quality assurance criteria for specialization schools $[5,6]$. However, the detailed definition of some learning objectives has been assigned to the individual local academic communities, thus leaving the basics for some teaching areas less precisely defined.

Therefore, the results of the present survey and the fact that not all the RT schools can rely on adequate volumes of procedures or on availability of experts for all the potential applications of BT, should be carefully considered. The opportunity of a geographically well-distributed network of learning sites organized by the residential schools has been therefore envisaged. A potential solution could also be to set up specific inter-University training courses (e.g., Masters) for ROs interested to acquire a specific expertise in BT.

These University-certified learning courses could also help to select BT professionals' services offered to health providers. Additionally, creating national/international technology transfer fellowships or supported attendance to national/international courses could serve as a part of a national educational programme.

\section{Conclusions}

This survey has evidenced the need for an improvement of interventional radiotherapy (BT) education in Italy, especially to better define and harmonize programs at national level, particularly in practical teaching. The ESTRO core curriculum could be considered as a sound basis for the creation of a national BT education programme, based on a geographic network for BT practical teaching. Supporting the attendance of national and international courses on specific aspects of BT may also facilitate the achievement of a satisfying level of BT knowledge among RO's. Finally, Universities may establish post-specialty courses ('second level' Masters) to allow professionals (already certified in radiation oncology) to acquire more complex BT knowledges. This could facilitate the recruitment of skilled professionals for hospitals' BT units.

\section{Acknowledgements}

We would like to thank Dr. Sara Pedretti (University and Spedali Civili Hospital, Brescia), Dr. Paola Vitali (University and Spedali Civili Hospital, Brescia) and Dr. Antonio Diroma (Università Cattolica del Sacro Cuore).

\section{Disclosure}

The authors report no conflict of interest.

\section{References}

1. Han K, Milosevic M, Fyles A et al. Trends in the utilization of brachytherapy in cervical cancer in the United States. Int J Radiation Oncol Biol Phys 2013; 87: 111-119.

2. Hoskin PJ, Bownes P. Innovative technologies in radiation therapy: brachytherapy. Semin Radiat Oncol 2006; 16: 209-217.

3. Eriksen JG, Beavis AW, Coffey MA et al. The updated ESTRO core curricula 2011 for clinicians, medical physicists and RTTs in radiotherapy/radiation oncology. Radiother Oncol 2012; 103: 103-108.

4. Autorino R, Vicenzi L, Tagliaferri L et al. A national survey of AIRO (Italian Association of Radiation Oncology) brachytherapy (Interventional Radiotherapy) study group. J Contemp Brachytherapy 2018; 10: 254-259.

5. MIUR - D.L. n. 402/2017 Standard, requisiti e indicatori di attivita' formativa e assistenziale delle Scuole di specializzazione di area sanitaria. (17A04639) (GU Serie Generale n.163 del 14-07-2017 - Suppl. Ordinario n. 38). http:/ / www. gazzettaufficiale.it/eli/id/2017/07/14/17A04639/sg

6. 14-7-2017 Supplemento ordinario n. 38 alla GAZZETTA UFFICIALE Serie generale - n. 163, Allegato 3. http://www. gazzettaufficiale.it/eli/gu/2017/07/14/163/so/38/sg/pdf

7. Tagliaferri L, Pagliara MM, Boldrini L et al. INTERACTS (INTErventional Radiotherapy ACtive Teaching School) guidelines for quality assurance in choroidal melanoma interventional radiotherapy (brachytherapy) procedures. J Contemp Brachytherapy 2017; 9: 287-295.

8. Kovács G, Tagliaferri L, Valentini V. Is an Interventional Oncology Center an advantage in the service of cancer patients or in the education? The Gemelli Hospital and INTERACTS experience. J Contemp Brachytherapy 2017; 9: 497-498.

9. Fellin G, Mirri MA, Santoro L et al. Low dose rate brachytherapy (LDR-BT) as monotherapy for early stage prostate cancer in Italy: practice and outcome analysis in a series of 2,237 patients from 11 institutions. Br J Radiol 2016; 89: 20150981.

10. Frakulli R, Buwenge M, Cammelli $S$ et al. Brachytherapy boost after chemoradiation in anal cancer: a systematic review. J Contemp Brachytherapy 2018; 10: 246-253.

11. Lancellotta V, Kovács G, Tagliaferri L et al. Age is not a limiting factor in interventional radiotherapy (brachytherapy) for patients with localized cancer. Biomed Res Int 2018; 2018: 2178469.

12. Autorino R, Vicenzi L, Tagliaferri L et al. A national survey of AIRO (Italian Association of Radiation Oncology) brachytherapy (Interventional Radiotherapy) study group. J Contemp Brachytherapy 2018; 10: 254-259.

13. Blasi MA, Laguardia M, Tagliaferri L et al. Brachytherapy alone or with neoadjuvant photodynamic therapy for amelanotic choroidal melanoma: functional outcomes and local tumor control. Retina 2016; 36: 2205-2212.

14. Frakulli R, Galuppi A, Cammelli $S$ et al. Brachytherapy in non-melanoma skin cancer of eyelid: a systematic review. J Contemp Brachytherapy 2015; 7: 497-502.

15. Tagliaferri L, Pagliara MM, Masciocchi C et al. Nomogram for predicting radiation maculopathy in patients treated with Ruthenium-106 plaque brachytherapy for uveal melanoma. J Contemp Brachytherapy 2017; 9: 540-547.

16. Tagliaferri L, Bussu F, Fionda B et al. Perioperative HDR brachytherapy for reirradiation in head and neck recurrences: single-institution experience and systematic review. Tumori 2017; 103: 516-524.

17. Aristei C, Maranzano E, Lancellotta V et al. Partial breast irradiation with interstitial multi-catheter high-dose-rate brachytherapy. Long-term results of a phase II prospective study. Radiother Oncol 2017; 124: 208-213.

18. Bussu F, Tagliaferri L, Mattiucci G et al. Comparison of interstitial brachytherapy and surgery as primary treatments for nasal vestibule carcinomas. Laryngoscope 2016; 126: 367-371.

19. Perrucci E, Lancellotta V, Bini V et al. Recurrences and toxicity after adjuvant vaginal brachytherapy in Stage I-II endometrial cancer: A monoinstitutional experience. Brachytherapy 2016; 15: 177-184

20. De Sanctis V, Agolli L, Valeriani M et al. External-beam radiotherapy and/or HDR brachytherapy in postoperative endometrial cancer patients: clinical outcomes and toxicity rates. Radiol Med 2013; 118: 311-322. 
21. Fuccio L, Mandolesi D, Farioli A et al. Brachytherapy for the palliation of dysphagia owing to esophageal cancer: A systematic review and meta-analysis of prospective studies. $R a-$ diother Oncol 2017; 122: 332-339.

22. Tagliaferri L, Bussu F, Rigante M et al. Endoscopy-guided brachytherapy for sinonasal and nasopharyngeal recurrences. Brachytherapy 2015; 14: 419-425.

23. Mattiucci GC, Autorino R, Tringali A et al. A phase I study of high-dose-rate intraluminal brachytherapy as palliative treatment in extrahepatic biliary tract cancer. Brachytherapy 2015; 14: 401-404.

24. Giannelli F, Chiola I, Belgioia L et al. Complete response in a patient with gynecological hidradenocarcinoma treated with exclusive external beam radiotherapy and brachytherapy: a case report. J Contemp Brachytherapy 2017; 9: 572-578.

25. Tagliaferri L, Manfrida S, Barbaro B et al. MITHRA - multiparametric MR/CT image adapted brachytherapy (MR/ CT-IABT) in anal canal cancer: a feasibility study. J Contemp Brachytherapy 2015; 7: 336-345.

26. Autorino R, Mattiucci GC, Ardito F et al. Radiochemotherapy with gemcitabine in unresectable extrahepatic cholangiocarcinoma: long-term results of a phase ii study. Anticancer Res 2016; 36: 737-740.

27. Fuccio L, Guido A, Hassan C et al. Underuse of brachytherapy for the treatment of dysphagia owing to esophageal cancer. An Italian survey. Dig Liver Dis 2016; 48: 1233-1236.

28. Cortesi A, Galuppi A, Frakulli R et al. Adjuvant radiotherapy with brachytherapy boost in soft tissue sarcomas. J Contemp Brachytherapy 2017; 9: 256-262.

29. Barbera F, Triggiani L, Buglione M et al. Salvage low dose rate brachytherapy for recurrent prostate cancer after external beam radiotherapy: results from a single institution with focus on toxicity and functional outcomes. Clin Med Insights Oncol 2017; 11: 1179554917738765.

30. Pagliara MM, Tagliaferri L, Azario L et al. Ruthenium brachytherapy for uveal melanomas: Factors affecting the development of radiation complications. Brachytherapy 2018; 17: 432-438.

31. Perrucci E, Lancellotta V, Bini V et al. Quality of life and cosmesis after breast cancer: whole breast radiotherapy vs partial breast high-dose-rate brachytherapy. Tumori 2015; 101: 161-167.

32. Haie-Meder C, Siebert FA, Pötter R. Image guided, adaptive, accelerated, high dose brachytherapy as model for advanced small volume radiotherapy. Radiother Oncol 2011; 100: 333-343.

33. Niehoff P, Dietrich J, Ostertag H et al. High-dose-rate (HDR) or pulsed-dose-rate (PDR) perioperative interstitial intensity-modulated brachytherapy (IMBT) for local recurrences of previously irradiated breast or thoracic wall following breast cancer. Strahlenther Onkol 2006; 182: 102-107.

34. Williamson JF. Current brachytherapy quality assurance guidance: does it meet the challenges of emerging image-guided technologies? Int J Radiat Oncol Biol Phys 2008; 71: S18-22.

35. Doniec JM, Schniewind B, Kovács G et al. Multimodal therapy of anal cancer added by new endosonographic-guided brachytherapy. Surg Endosc 2006; 20: 673-678.

36. Kirchheiner K, Nout RA, Tanderup K et al. Manifestation pattern of early-late vaginal morbidity after definitive radiation (chemo)therapy and image-guided adaptive brachytherapy for locally advanced cervical cancer: an analysis from the EMBRACE study. Int J Radiat Oncol Biol Phys 2014; 89: 88-95.

37. Kirisits C, Federico M, Nkiwane $\mathrm{K}$ et al. Quality assurance in MR image guided adaptive brachytherapy for cervical cancer: Final results of the EMBRACE study dummy run. Radiother Oncol 2015; 117: 548-554.
38. Nkiwane KS, Pötter R, Tanderup K et al. Single line source with and without vaginal loading and the impact on target coverage and organ at risk doses for cervix cancer Stages IB, II, and IIIB: treatment planning simulation in patients treated with MRI-guided adaptive brachytherapy in a multicentre study (EMBRACE). Brachytherapy 2013; 12: 317-323.

39. Pötter R, Tanderup K, Kirisits $C$ et al. The EMBRACE II study: The outcome and prospect of two decades of evolution within the GEC-ESTRO GYN working group and the EMBRACE studies. Clin Transl Radiat Oncol 2018; 9: 48-60.

40. Sturdza A, Pötter R, Fokdal LU et al. Image guided brachytherapy in locally advanced cervical cancer: Improved pelvic control and survival in RetroEMBRACE, a multicenter cohort study. Radiother Oncol 2016; 120: 428-433.

41. Fokdal L, Sturdza A, Mazeron R et al. Image guided adaptive brachytherapy with combined intracavitary, and interstitial technique improves the therapeutic ratio in locally advanced cervical cancer: Analysis from the retroEMBRACE study. Radiother Oncol 2016; 120: 434-440.

42. Rodda S, Morris WJ, Hamm J et al. ASCENDE-RT: An analysis of health-related quality of life for a randomized trial comparing low-dose-rate brachytherapy boost with dose-escalated external beam boost for high- and intermediate-risk prostate cancer. Int J Radiat Oncol Biol Phys 2017; 98: 581-589.

43. Rodda S, Tyldesley S, Morris WJ et al. ASCENDE-RT: An analysis of treatment-related morbidity for a randomized trial comparing a low-dose-rate brachytherapy boost with a dose-escalated external beam boost for high- and intermediate-risk prostate cancer. Int J Radiat Oncol Biol Phys 2017; 98: 286-295.

44. Polgár C, Van Limbergen E, Pötter R et al. GEC-ESTRO breast cancer working group. Patient selection for accelerated partial-breast irradiation (APBI) after breast-conserving surgery: recommendations of the Groupe Européen de Curiethérapie-European Society for Therapeutic Radiology and Oncology (GEC-ESTRO) breast cancer working group based on clinical evidence (2009). Radiother Oncol 2010; 94: 264-273.

45. Armpilia C, Dale RG, Sandilos P et al. Radiobiological modelling of dose-gradient effects in low dose rate, high dose rate and pulsed brachytherapy. Phys Med Biol 2006; 51: 4399-4411.

46. Joslin CAF, Flynn A, Hall EJ. Principles and practice of brachytherapy using afterloading systems. London, Arnold, 2001.

47. Mould RF, Batterman JJ, Martinez AA et al. Brachytherapy from radium to optimization. Nucletron International BV, Veenendaal, The Netherlands 1994.

48. Lievens Y, De Schutter H, Stellamans KP et al. Radiotherapy access in Belgium: How far are we from evidence-based utilization? Eur J Cancer 2017; 84: 102-113. 